\title{
Robust Fault Detection for LPV Systems using Interval Observers and Zonotopes
}

\author{
Fatiha Nejjari, Vicenç Puig, Saúl Montes de Oca and Atefeh Sadeghzadeh
}

\begin{abstract}
In this paper, the problem of robust fault detection using an interval observer for dynamic systems characterized by LPV (Linear Parameter Varying) models is presented. The observer faces the robustness problem using two complementary strategies. Modeling uncertainties are considered unknown but bounded by intervals. Their effect is addressed using an interval state observation method based on zonotope representation of the set of possible states. The observer gain is designed via pole placement using LMI formulation. The method is applied to a LPV representation of a Twin Rotor MIMO System.

Index Terms-Robust Fault Detection, Linear Parameter Varying, Interval LPV Observer, Linear Matrix Inequalities, Zonotopes.
\end{abstract}

\section{INTRODUCTION}

Model-based fault detection and isolation (FDI) for dynamic systems have been an active research field these last decades.

Many model-based FDI techniques, mostly based on linear models, have been investigated and developed in the literature over the last few years. The use of FDI linearbased methods has been extended to non-linear systems using linearization around an operating point [1]. However, for systems with high non-linearity and a wide operating range, the linearized approach fails to give satisfactory results. To tackle this problem, new fault detection methods based on non-linear models have been developed. Methods range from the direct use of non-linear models to the use of neural networks, TS fuzzy systems and neuro-fuzzy systems [1].

Alternatively, Linear Parameter Varying (LPV) models have recently attracted the attention of the FDI research community. Such models can be used efficiently to represent some nonlinear systems [2], [3]. This has motivated some researchers from the FDI community to develop model-based methods using LPV models (see [4], among others). But even with the use of LPV models, modeling errors and disturbances are inevitable in complex engineering systems. So, in order to increase reliability and performance of model-based fault detection, the development of robust fault detection algorithms should be addressed. The robustness of a fault detection system means that it must be only sensitive to faults, even in the presence of model-reality differences [1]. One of the approaches to robustness, known as passive, is based on enhancing the robustness of the fault detection system at the decision-making stage. The aim with the passive approach is usually to determine, given a set of

F. Nejjari, V. Puig, S. Montes de Oca and A. Sadeghzadeh are with Automatic Control Department, Universitat Politècnica de Catalunya (UPC), Rambla de Sant Nebridi, 11, 08222 Terrassa, Spain. e-mail: vicenc.puig@upc.edu models, if there is any member in the set that can explain the measurements. A common approach to this problem is to propagate the model uncertainty to the alarm limits of the residuals. When the residuals are outside of the alarm limits, it is argued that model uncertainty alone can not explain the residual and therefore a fault must have occurred. This approach has the drawback that faults that produce a residual deviation smaller than the residual uncertainty due to parameter uncertainty will not be detected. Another approach to the passive robust fault detection problem is to explicitly calculate the set of states that are consistent with the measurements. When a measurement is found to be inconsistent with this set, a fault is assumed to have occurred. As an exact representation of the set of states consistent with the measurements is hard to calculate, approximating sets that provide outer bounds are often used instead.

In the literature, several approximating sets to enclose the set of possible states have been proposed. In [5], a state estimator based on enclosing the set of states by the smallest ellipsoid is proposed following the algorithms proposed by [6]. However, in this approach only additive uncertainty is considered, but not the multiplicative one introduced by modeling uncertainty located in the parameters. In this paper, both types of uncertainties are considered as in [7], but there only system trajectories obtained from the uncertain parameter interval vertices are considered, assuming that the monotonicity property holds.

The main contribution of this paper is to develop a passive robust fault method for LPV systems that uses an interval observer approach based on enclosing the set of states by zonotopes. The proposed state-estimator applied to fault detection follows a consistency based approach that is based on determining the set of states that are consistent with parameter and measurement uncertainty.

\section{INTERVAL OBSERVERS FOR LPV SYSTEMS}

\section{A. System set-up}

Let us consider that the nonlinear system to be monitored can be described by its LPV representation as follows:

$$
\begin{aligned}
x(k+1) & =A\left(\tilde{\vartheta_{k}}\right) x(k)+B\left(\tilde{\vartheta_{k}}\right) u(k) \\
y(k) & =C\left(\tilde{\vartheta_{k}}\right) x(k)+D\left(\tilde{\vartheta_{k}}\right) u(k)
\end{aligned}
$$

where $u(k) \in \Re^{n_{u}}$ is the system input, $y(k) \in \Re^{n_{y}}$ is the system output and $x(k) \in \Re^{n_{x}}$ is the state-space vector. $\tilde{\vartheta}_{k}:=\vartheta(\tilde{)})$ is a vector of time-varying parameters of dimension $n_{\vartheta}$ that changes with the operating point scheduled by some measured system variables $p_{k}\left(p_{k}:=\right.$ $p(k))$ that can be estimated using some known function: 
$\vartheta_{k}=f\left(p_{k}\right)^{1}$. However, some uncertainty in the estimated values is considered to be bounded by:

$$
\Theta_{k}=\left\{\vartheta_{k} \in \Re^{n_{\vartheta}} \mid \quad \underline{\vartheta_{k}} \leq \vartheta_{k} \leq \overline{\vartheta_{k}}\right\}, \quad \vartheta_{k}=f\left(p_{k}\right)
$$

This set represents the uncertainty about the exact knowledge of real system parameters $\vartheta_{k}$. The interval for uncertain parameters can be inferred from real data using set-membership parameter estimation algorithms [8].

System (1) describes a model parameterized by a scheduling variable denoted by $p_{k}$. In this paper, the kind of LPV system considered are those whose parameters vary affinely in a polytope [9]. In particular, the state-space matrices range in a polytope of matrices defined as the convex hull of a finite number, $N$, of matrices. That is,

$$
\begin{gathered}
\left(\begin{array}{cc}
A\left(\tilde{\vartheta}_{k}\right) & B\left(\tilde{\vartheta}_{k}\right) \\
C\left(\tilde{\vartheta_{k}}\right) & D\left(\tilde{\vartheta_{k}}\right)
\end{array}\right) \in C o\left\{\left(\begin{array}{cc}
A_{j}\left(\vartheta^{j}\right) & B_{j}\left(\vartheta^{j}\right) \\
C_{j}\left(\vartheta^{j}\right) & D_{j}\left(\vartheta^{j}\right)
\end{array}\right)\right\} \\
:=\sum_{j=1}^{N} \alpha^{j}\left(p_{k}\right)\left(\begin{array}{ll}
A_{j}\left(\vartheta^{j}\right) & B_{j}\left(\vartheta^{j}\right) \\
C_{j}\left(\vartheta^{j}\right) & D_{j}\left(\vartheta^{j}\right)
\end{array}\right)
\end{gathered}
$$

with $\alpha^{j}\left(p_{k}\right) \geq 0, \sum_{j=1}^{N} \alpha^{j}\left(p_{k}\right)=1$ and $\vartheta^{j}=f\left(p^{j}\right)$ is the vector of uncertain parameters of $j^{\text {th }}$ model where each $j^{\text {th }}$ model is called a vertex system and it is assumed according to property (2) that: $\vartheta^{j} \in\left[\underline{\vartheta^{j}}, \overline{\vartheta^{j}}\right]$.

Consequently, the LPV system (1) can be expressed as follows:

$$
\begin{aligned}
x(k+1) & =\sum_{j=1}^{N} \alpha^{j}\left(p_{k}\right)\left[A_{j}\left(\vartheta^{j}\right) x(k)+B_{j}\left(\vartheta^{j}\right) u(k)\right] \\
y(k) & =\sum_{j=1}^{N} \alpha^{j}\left(p_{k}\right)\left[C_{j}\left(\vartheta^{j}\right) x(k)+D_{j}\left(\vartheta^{j}\right) u(k)\right]
\end{aligned}
$$

Here $A_{j}, B_{j}, C_{j}$ and $D_{j}$ are the state space matrices corresponding to $j^{\text {th }}$ model. Notice that, the state space matrices of system (1) is equivalent to the interpolation between LTI models, for example: $A\left(\tilde{\vartheta}_{k}\right)=\sum_{j=1}^{N} \alpha^{j}\left(p_{k}\right) A_{j}\left(\vartheta^{j}\right)$.

The polytopic system is scheduled through functions designed as follows: $\alpha^{j}\left(p_{k}\right), \forall j \in[1, \ldots, N]$ that lie in a convex set:

$$
\begin{gathered}
\Psi=\left\{\alpha^{j}\left(p_{k}\right) \in \mathbb{R}^{N}, \alpha\left(p_{k}\right)=\left[\alpha^{1}\left(p_{k}\right), \ldots, \alpha^{N}\left(p_{k}\right)\right]^{T}\right. \\
\left.\alpha^{j}\left(p_{k}\right) \geq 0, \forall j, \sum_{j=1}^{N} \alpha^{j}\left(p_{k}\right)=1\right\}
\end{gathered}
$$

There are several ways of implementing (3) depending on how $\alpha^{j}\left(p_{k}\right)$ functions are defined [10]. Here the approach used in [11] is proposed:

$$
\begin{aligned}
& \left(\begin{array}{cc}
A\left(\tilde{\vartheta}_{k}\right) & B\left(\tilde{\vartheta}_{k}\right) \\
C\left(\tilde{\vartheta}_{k}\right) & D\left(\tilde{\vartheta_{k}}\right)
\end{array}\right) \\
& =\sum_{j=1}^{N} \underbrace{\sum_{i_{1}=1}^{2} \cdots \sum_{i_{v}=1}^{2} \prod_{m=1}^{v} \mu_{m, i_{m}}\left(p_{m}(k)\right)}_{\alpha^{j}\left(p_{k}\right)}\left(\begin{array}{cc}
A_{j}\left(\vartheta^{j}\right) & B_{j}\left(\vartheta^{j}\right) \\
C_{j}\left(\vartheta^{j}\right) & D_{j}\left(\vartheta^{j}\right)
\end{array}\right)
\end{aligned}
$$

\footnotetext{
${ }^{1}$ Note that the $\vartheta(k)$ denotes the real system vector of time-varying parameters and $\vartheta(k)$ represents the estimated value
}

with $\mu_{m, 1}=\frac{\left(p_{m}(k)-\underline{p_{m}^{j}}\right)}{\left(\overline{p_{m}^{j}}-\underline{p_{m}^{j}}\right)}$ and $\mu_{m, 2}=1-\mu_{m, 1}$ where $\overline{p_{m}^{j}}$ and $p_{m}^{j}$ represent the upper and lower bounds of $p_{m}$ respectively $\overline{\text { and }} v$ is the number of scheduling variables.

The system described by (1) is monitored using a linear observer with Luenberger structure considering parameter uncertainty given by $\vartheta^{j} \in\left[\underline{\vartheta^{j}}, \overline{\vartheta^{j}}\right]$. In the following, we consider only strictly proper systems such that $D=0$. Consequently, the interval LPV observer can be written as:

$$
\begin{aligned}
\hat{x}(k+1) & =\sum_{j=1}^{N} \alpha^{j}\left(p_{k}\right)\left[A_{0, j}\left(\vartheta^{j}\right) \hat{x}(k)+B_{j}\left(\vartheta^{j}\right) u(k)+L_{j} y(k)\right] \\
\hat{y}(k) & =\sum_{j=1}^{N} \alpha^{j}\left(p_{k}\right)\left[C_{j}\left(\vartheta^{j}\right) \hat{x}(k)\right]
\end{aligned}
$$

where $A_{0, j}\left(\vartheta^{j}\right)=A_{j}\left(\vartheta^{j}\right)-L_{j} C_{j}\left(\vartheta^{j}\right), u(k)$ is the measured system input vector, $\hat{x}(k)$ is the estimated system state vector, $\hat{y}(k)$ is the estimated system output vector and $L_{j}$ is the observer gain that has to be designed in order to stabilize the observer given by (7) for all $\vartheta^{j} \in\left[\underline{\vartheta}^{j}, \bar{\vartheta}^{j}\right]$.

Definition 1. Consider the state estimator given by (7), an initial compact set $\mathbb{X}_{0}$ and a sequence of measured inputs $(u(i))_{0}^{k-1}$ and outputs $(y(i))_{0}^{k}$. The exact uncertain estimated state set $\mathbb{X}_{k}$ and the output set $\mathbb{Y}_{k}$ at time $k$ are:

$$
\begin{gathered}
\mathbb{X}_{k}, \mathbb{Y}_{k}=\left\{\hat{x}_{k}, \hat{y}_{k}:\left(\hat{x}(i)=\sum_{j=1}^{N} \alpha^{j}\left(p_{i}\right)\left[A_{j}\left(\vartheta^{j}\right) \hat{x}(i-1)\right.\right.\right. \\
\left.\left.+B_{j}\left(\vartheta^{j}\right) u(i-1)+L_{j}\left(\vartheta^{j}\right)(y(i-1)-\hat{y}(i-1))\right]\right)_{i=1}^{k} \\
\left.\left(\hat{y}(i-1)=\sum_{j=1}^{N} \alpha^{j}\left(p_{i}\right) C_{j}\left(\vartheta^{j}\right) \hat{x}(i-1)\right)_{i=1}^{k} \mid \hat{x}_{0} \in \mathbb{X}_{0}\right\}
\end{gathered}
$$

for all $\vartheta_{k} \in \Theta_{k}$.

The uncertain state set described in Definition 1 at time $k$ can be computed approximately by admitting the rupture of the existing relations between variables of consecutive time instants. This makes possible to compute an approximation of this set from the approximate uncertain state set at time $k-1$.

Definition 2. Consider a system given by (7), a set of uncertain states/outputs at time $k-1\left(\mathbb{X}_{k-1}, \mathbb{Y}_{k-1}\right)$ and the input/ouput values $\left(u_{k-1}, y_{k-1}\right)$. Then, the approximated set of estimated states $\mathbb{X}_{k}^{e}$ and outputs $\mathbb{Y}_{k}^{e}$ at time $k$ based on and the measurements up to time $k-1$ are defined as:

$$
\begin{array}{r}
\mathbb{X}_{k}^{e}, \mathbb{Y}_{k}^{e}=\left\{\hat{x}_{k}, \hat{y}_{k}: \hat{x}(k)=\sum_{j=1}^{N} \alpha^{j}\left(p_{k}\right)\left[A_{j}\left(\vartheta^{j}\right) \hat{x}(k-1)\right.\right. \\
\left.+B_{j}\left(\vartheta^{j}\right) u(k-1)+L_{j}\left(\vartheta^{j}\right)(y(k-1)-\hat{y}(k-1))\right] \\
\left.\hat{y}(k-1)=\sum_{j=1}^{N} \alpha^{j}\left(p_{k}\right) C_{j}\left(\vartheta^{j}\right) \hat{x}(k-1) \mid \hat{x}_{0} \in \mathbb{X}_{0}\right\}
\end{array}
$$

for all $\vartheta_{k} \in \Theta_{k}$.

Since the set of estimated states $\mathbb{X}_{k}^{e}$ and estimated outputs $\mathbb{Y}_{k}^{e}$ is difficult to compute, one way is to bound it using some geometric regions easy to compute as for example: a box (interval hull) as in [12], an ellipsoid as in [6] or a zonotope as in [13].

Here, the set of estimated states $\mathbb{X}_{k}^{e}$ (or outputs $\mathbb{Y}_{k}^{e}$ ) introduced in Definition 2 will be approximated iteratively 
using zonotopes. From these zonotopes, an interval for each state variable can also be obtained by computing the interval hull of the zonotope. The sequence of interval hulls $\square \mathbb{X}_{k}^{e}$ with $k \in[0, N]$ will be called the interval LPV observer estimation of the system given by (7). Analogously, the sequence of interval hulls $\square \mathbb{Y}_{k}^{e}$ can be obtained. Following the previous idea, Algortihm $l$ is proposed to determine an approximation of set of uncertain estimated states.

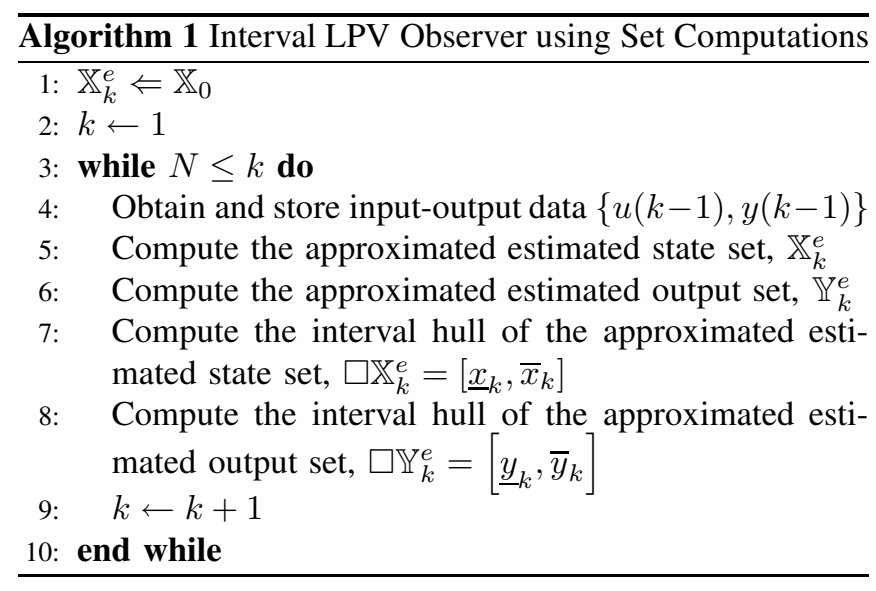

\section{B. Observer design}

The design of the interval LPV observer (7) can be solved with the LMI pole placement technique [14], [15], that allows to locate the poles of the observer in a subregion of the left half-plane using a LMI region.

In particular, let consider a disk LMI region called $\mathcal{D}$ defined by an affix $(-a, 0)$ and a radius $r$ such that $(a+$ $r)<1$. The two scalars $a$ and $r$ are used to determine a specific region included in the unit circle where the observer eigenvalues will be placed. Therefore, this circular region puts a lower bound on both the exponential decay rate and the damping ratio of the closed-loop response. The design of the interval LPV observer (7) such that the observer poles are placed in this LMI region requires to find for each vertex $j^{\text {th }}$ (with $j \in[1, \ldots, N]$ ) the observer gain $L_{j}$ and unknown symmetric matrix $X_{j}=X_{j}^{T}>0$ that satisfies the following LMI :

$$
\left(\begin{array}{cc}
-r X_{j} & a X_{j}+\left(A_{0, j}\left(\vartheta^{j}\right)^{T} X_{j}\right)^{T} \\
\left(a+A_{0, j}\left(\vartheta^{j}\right)^{T}\right) X_{j} & -r X_{j}
\end{array}\right)<0,
$$

for $\vartheta^{j} \in\left[\underline{\vartheta^{j}}, \overline{\vartheta^{j}}\right]$.

Note that expression (10) is a Bilinear Matrix Inequality (BMI) which cannot be solved with LMI classical tools. By substituting $W_{j}=L_{j}^{T} X_{j}$, it is possible to transform it into:

$$
\left[\begin{array}{cc}
-r X_{j} & \cdots \\
\left(a+A_{0, j}\left(\vartheta^{j}\right)^{T}\right) X_{j}-C_{j}\left(\vartheta^{j}\right)^{T} W_{j} & \cdots \\
a X_{j}+X_{j}^{T} A_{0, j}\left(\vartheta^{j}\right)-W_{j}^{T} C_{j}\left(\vartheta^{j}\right) & -r X_{j}
\end{array}\right]<0 .
$$

Then, the design procedure boils down to solving the LMI (11) and then determining $L_{j}=\left(W_{j} X_{j}^{-1}\right)^{T}$. Finally, the observer gains $L_{j}$ will be interpolated to obtain the interval LPV observer (7) as: $L=\sum_{j=1}^{N} \alpha^{j}\left(p_{k}\right) L_{j}$.

\section{Fault detection using interval LPV observers}

The application of observers to fault detection consists in testing whether the measured output is consistent with the one given by an observer using a faultless model. If an inconsistency is detected, the existence of a fault is proved. In case of modeling a dynamic system using an interval model, the predicted output $\hat{y}(k)$ is described by a set that can be bounded using an interval. Then, the fault detection test can be stated as:

$$
y(k) \notin \square \mathbb{Y}_{k}^{e}
$$

where $\mathbb{Y}_{k}^{e}$ is the set of predicted outputs that can be obtained using Algorithm 1 and $\square \mathbb{Y}_{k}^{e}=\left[\underline{y}_{1}, \bar{y}_{1}\right] \times \cdots \times\left[\underline{y}_{n_{y}}, \bar{y}_{n_{y}}\right]$.

Alternatively, a fault detection based on generating a residual can be used. The residual is generated by comparing the measurements of physical variables $y(k)$ of the process with their estimation $\hat{y}(k)$ provided by the associated system model:

$$
r(k)=y(k)-\hat{y}(k)
$$

where $r(k) \in \Re^{n_{y}}$ is the residual set. Then, the fault detection test can be expressed as follows:

$$
r(k) \notin \square \mathbb{R}_{k}
$$

where $\mathbb{R}_{k}$ is the set of residuals that can be calculated with the expression (13) and $\square \mathbb{R}_{k}=\left[\underline{r}_{1}, \bar{r}_{1}\right] \times \cdots \times\left[\underline{r}_{n_{y}}, \bar{r}_{n_{y}}\right]$.

Algorithm 2 implements fault detection using interval observers and the fault detection test presented in (12).

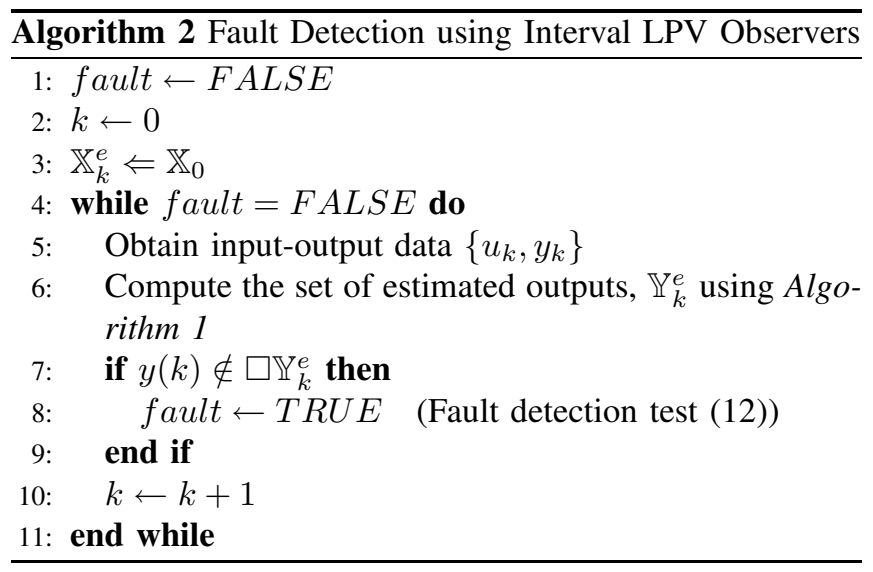

Note that the fault detection test in Algorithm 1 could also have been implemented using (14).

\section{IMPLEMENTATION USING ZONOTOPES}

\section{A. Introduction}

In this paper, zonotopes are used to bound the set of uncertain estimated sets. Let us introduce zonotopes.

Definition 3. The Minkowski sum of two sets $\mathbb{X}$ and $\mathbb{Y}$ is defined by $\mathbb{X} \oplus \mathbb{Y}=\{x+y: x \in \mathbb{X}, y \in \mathbb{Y}\}$.

Definition 4. Given a center vector $\pi \in \mathbb{R}^{n}$ and a matrix $\mathrm{H} \in \mathbb{R}^{n \times m}$ the Minkowski sum of the segments defined by 
the columns of matrix $\mathrm{H}$, is called a zonotope of order $m$. This set is represented as:

$$
\mathbb{X}=\pi \oplus H \beta^{m}=\left\{\pi+H z: z \in \beta^{m}\right\}
$$

where: $\beta^{m}$ is a unitary box, composed by $\mathrm{m}$ unitary intervals.

Definition 5. The interval hull $\square \mathbb{X}$ of a closed set $\mathbb{X}$ is the smallest interval box that contains $\mathbb{X}$.

Given a zonotope $\mathbb{X}=\pi \oplus H \beta^{m}$, its interval hull can be easily computed by evaluating $\pi \oplus H \beta^{m}$, for all $i=1, \ldots, n$ : $\square \mathbb{X}=\left\{x:\left|x_{i}-\pi_{i}\right| \leq\left\|H_{i}\right\|_{1}\right\}$ where $H_{i}$ is $i^{\text {th }}$-row of $H$, and $x_{i}$ and $\pi_{i}$ are $i^{t h}$ components of $x$ and $\pi$, respectively.

\section{B. Implementation of interval LPV observers using zono-} topes

To implement interval LPV observers using zonotopes, it should be noticed that using (7) as the expression of the estimator model, it can be viewed as a discrete-time system with one input that can be reorganized as:

$$
\begin{aligned}
\hat{x}(k+1) & =\sum_{j=1}^{N} \alpha^{j}\left(p_{k}\right)\left[A_{0, j}\left(\vartheta^{j}\right) \hat{x}(k)+B_{0, j}\left(\vartheta^{j}\right) v(k)\right] \\
\hat{y}(k) & =\sum_{j=1}^{N} \alpha^{j}\left(p_{k}\right) C_{j}\left(\vartheta^{j}\right) \hat{x}(k)
\end{aligned}
$$

where: $B_{0, j}\left(\vartheta^{j}\right)=\left[B_{j}\left(\vartheta^{j}\right) L_{j}\right], v_{(k)}=\left[\begin{array}{ll}u_{k} & y_{k}\end{array}\right]^{T}$ and $A_{0, j}\left(\vartheta^{j}\right)=A_{0, j}\left(\vartheta^{j}\right)-L_{j} C_{j}\left(\vartheta^{j}\right)$.

Then, the problem of interval observation can be formulated as a problem of interval simulation and requires characterizing the set $\mathbb{X}_{k}^{e}$. This set can be viewed as the direct image evaluation of (15) and can be implemented using zonotopes.

According to Algorithm 1, interval LPV observers involves a bounding operation applied to the set of estimated states $\mathbb{X}_{k}^{e}$.

\section{Implementation of prediction set step}

The prediction set step requires characterizing the set $\mathbb{X}_{k}^{e}$. This set can be viewed as the direct image evaluation of $f\left(x_{k}, \vartheta_{k}\right)=\sum_{j=1}^{N} \alpha^{j}\left(p_{k}\right)\left[A_{0, j}\left(\vartheta^{j}\right) \hat{x}(k)+B_{0, j}\left(\vartheta^{j}\right) v(k)\right]$. There are different algorithms to bound such an image using ellipsoids (see [6]) or zonotopes (see [16]). To bound such image using zonotopes the following result is used:

Theorem 1. "Zonotope Inclusion" (see [13]). Consider a family of zonotopes represented by $\mathbb{X}=\pi \oplus M \beta^{m}$ where $\pi \in \mathbb{R}^{n}$ is a real vector and $M \in \mathbb{I}^{n \times m}$ is an interval matrix. A zonotope inclusion $\diamond(\mathbb{X})$ is defined by:

$$
\diamond(\mathbb{X})=\pi \oplus[\operatorname{mid}(M \quad G)]\left[\begin{array}{c}
\beta^{m} \\
\beta^{n}
\end{array}\right]=\pi \oplus J \beta^{n+m}
$$

where $G \in \mathbb{R}^{n \times n}$ is a diagonal matrix that satisfies: $G_{i i}=$ $\sum_{j=1}^{m} \frac{\operatorname{diam}\left(M_{i j}\right)}{2}, i=1,2, \ldots, n$, mid denotes the center and diam the diameter of the interval according to [17]. Under this definition, $\mathbb{X} \subseteq \diamond(\mathbb{X})$.

This prediction step aims at computing the zonotope $\mathbb{X}_{k+1}^{e}$ that bounds the trajectory of the system at instant $k+1$, from the previous approximating zonotope at time instant $k, \mathbb{X}=$ $\pi(k) \oplus H_{k} \beta^{m}$, using the natural interval extension of (15) as suggested by [17] and the zonotope inclusion operator, as a generalization of Kühn's method (see [16]):

$$
\mathbb{X}_{k+1}^{e}=\pi(k+1) \oplus H_{k+1} \beta^{r}
$$

where:

$$
\begin{aligned}
\pi_{k+1} & =\operatorname{mid}\left(A_{0}\left(\vartheta_{k}\right)\right) \pi_{k}+\operatorname{mid}\left(B_{0}\left(\vartheta_{k}\right)\right) u_{k}^{o} \\
H_{k+1} & =\left[\begin{array}{lll}
J_{1} & J_{2} & J_{3}
\end{array}\right] \\
J_{1} & =\diamond\left(A_{0}\left(\vartheta_{k}\right) H_{k}\right) \\
J_{2} & =\left(\operatorname{diam}\left(A_{o}\left(\vartheta_{k}\right)\right) / 2\right) \pi(k) \\
J_{3} & =\left(\operatorname{diam}\left(B_{o}\left(\vartheta_{k}\right)\right) / 2\right) v(k)
\end{aligned}
$$

where $A_{0}=\sum_{j=1}^{N} \alpha^{j}\left(p_{k}\right) A_{0, j}\left(\vartheta^{j}\right), \quad B_{0}=$ $\sum_{j=1}^{N} \alpha^{j}\left(p_{k}\right) B_{0, j}\left(\vartheta^{j}\right)$ and $J_{1}$ is calculated using the zonotope inclusion operator.

It is important to notice that the set of estimated states has an increasing number of segments generating the zonotope $\mathbb{X}_{k+1}^{e}$ using this method. In order to control the domain complexity, a reduction step is thus implemented. Here we use the method proposed in [18] to reduce the zonotope complexity.

\section{CASE STUdy}

\section{A. Description of Twin-Rotor MIMO System}

The twin-rotor MIMO system (TRMS) is a laboratory setup developed by Feedback Instruments Limited for control experiments. The system is perceived as a challenging engineering problem due to its high non-linearity, cross-coupling between its two axes, and inaccessibility of some of its states through measurements. The TRMS mechanical unit has two rotors placed on a beam together with a counterbalance whose arm with a weight at its end is fixed to the beam at the pivot and it determines a stable equilibrium position (Fig.1). The TRMS consists of a beam pivoted on its base in such a way that it can rotate freely both in the horizontal and vertical planes. At both ends of the beam there are rotors (the main and tail rotors) driven by DC motors.

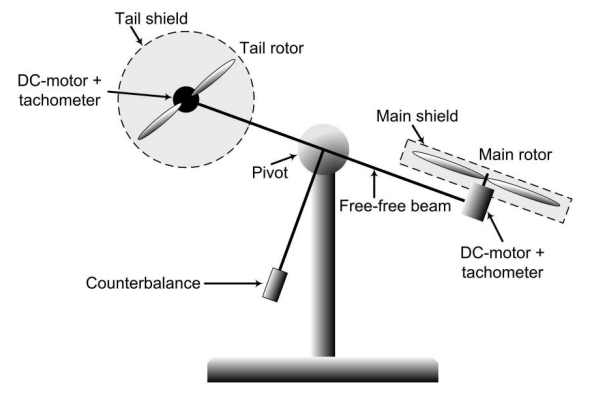

Fig. 1: Components of the Twin Rotor MIMO System

The system input vector is $u=\left[u_{t}, u_{m}\right]^{T}$ where $u_{t}$ is the input voltage of the tail motor and $u_{m}$ is the input voltage of the main motor. On the other hand, the system states are $x=\left[\theta_{h}, \Omega_{h}, \omega_{t}, \theta_{v}, \Omega_{v}, \omega_{m}\right]^{T}$ where $\Omega_{h}$ is the angular velocity around the vertical axis, $\theta_{h}$ is the azimuth angle of beam (horizontal plane), $\omega_{t}$ is the rotational velocity of the 
tail rotor, $\Omega_{v}$ is the angular velocity around the horizontal axis, $\theta_{v}$ is the pitch angle of beam (vertical plane) and $\omega_{m}$ is the rotational velocity of the main rotor.

\section{B. The TRMS LPV model and observer design}

LPV models can be obtained using state transformation, function substitution and methods using Jacobian linearization ([2], [10], [11], [19] and among others). In this case, the multiple linear model identification is used around different points (see Table 1) with a sampling time $T_{s}=0.025 \mathrm{~s}$. Then a global multi-model is obtained by interpolating among the local LTI models [10], [11]. The system has been identified using the input $u=\left[u_{t}, u_{m}\right]^{T}$ and the output $y=\left[\theta_{h}, \theta_{v}\right]^{T}$. Consider a system described by $N=5$ models, these five models should be adapted as a LPV model (7) where the matrices are:

$$
\begin{aligned}
A_{1}= & {\left[\begin{array}{cccccc}
1 & 0.025 & 0 & 0 & 0 & 0.0142 \\
0 & 0.9905 & 0 & 0 & 0.0995 & -0.0054 \\
0 & 0 & 1 & 0.025 & 0.0732 & 0 \\
0 & 0 & -0.0862 & 0.9976 & -0.0071 & 0.0078 \\
0 & 0 & 0 & 0 & 0.9349 & 0 \\
0 & 0 & 0 & 0 & 0 & 0.9825
\end{array}\right], } \\
A_{2}= & {\left[\begin{array}{ccccccc}
1 & 0.025 & -0.0005 & 0 & 0 & 0.0141 \\
0 & 0.9906 & 0.0002 & 0 & 0.0991 & -0.0053 \\
0 & 0 & 1 & 0.025 & 0.0732 & 0 \\
0 & 0 & -0.0862 & 0.9976 & -0.0071 & 0.0102 \\
0 & 0 & 0 & 0 & 0.9349 & 0 \\
0 & 0 & 0 & 0 & 0 & 0.9825
\end{array}\right], } \\
A_{5}= & {\left[\begin{array}{ccccccc}
1 & 0.025 & -0.0010 & 0 & 0 & 0.0137 \\
0 & 0.9908 & 0.0004 & 0 & 0.0985 & -0.0050 \\
0 & 0 & 1 & 0.025 & 0.0732 & 0 \\
0 & 0 & -0.0862 & 0.9976 & -0.0071 & 0.0207 \\
0 & 0 & 0 & 0 & 0.9349 & 0 \\
0 & 0 & 0 & 0 & 0 & 0.9825
\end{array}\right] } \\
A_{4}= & {\left[\begin{array}{ccccccc}
1 & 0.025 & -0.0015 & 0 & 0 & 0.0131 \\
0 & 0.9912 & 0.0005 & 0 & 0.0973 & -0.0046 \\
0 & 0 & 1 & 0.025 & 0.0732 & 0 \\
0 & 0 & -0.0862 & 0.9976 & -0.0071 & 0.0375 \\
0 & 0 & 0 & 0 & 0.9349 & 0 \\
0 & 0 & 0 & 0 & 0 & 0.9825 \\
0 & 0.9918 & 0.0006 & 0 & 0.0953 & -0.0040 \\
0 & 0 & 1 & 0.025 & 0.0732 & 0 \\
0 & 0 & -0.0860 & 0.9976 & -0.0071 & 0.0575 \\
0 & 0 & 0 & 0 & 0.9349 & 0 \\
0 & 0 & 0 & 0 & 0 & 0.9825
\end{array}\right], }
\end{aligned}
$$

where the scheduling variable is the azimuth angle of beam $\theta_{h}$ and $\alpha^{j}\left(p_{k}\right)$ can be determined from (6).

Table 1. Equilibrium points of the each $j^{t h}$ linear model.

\begin{tabular}{|c|c|c|c|c|}
\hline$N$ & $\bar{u}_{t}$ & $\bar{u}_{m}$ & $\bar{\theta}_{h}$ & $\bar{\theta}_{v}$ \\
\hline \hline 1 & 0 & 0 & 0 & -0.9326 \\
\hline 2 & 0 & 0.05 & 0.1074 & -0.9257 \\
\hline 3 & 0 & 0.10 & 0.2146 & -0.9133 \\
\hline 4 & 0 & 0.15 & 0.3199 & -0.8895 \\
\hline 5 & 0 & 0.20 & 0.4211 & -0.8501 \\
\hline
\end{tabular}

The LTI systems are incremental and should be added the equilibrium conditions of Table 1. Consequently, the expressions of these conditions are:

$$
\hat{y}(k)=\sum_{j=1}^{N} \alpha^{j}\left(\vartheta_{k}\right)\left[C_{j} \hat{x}(k)+\bar{y}_{j}\right]
$$

where $\bar{y}_{j}=\left[\bar{\theta}_{h}^{j}, \bar{\theta}_{v}^{j}\right]^{T}$ (See Table 1$)$.

Additionally, uncertainty has been included in some parameters of the observer model to taking into account the difference between the LPV model and the real nonlinear behavior: $a_{22}^{j} \in\left[a_{22}^{j} \pm 0.0092\right], a_{43}^{j} \in\left[a_{43}^{j} \pm 0.0133\right]$ and $a_{46}^{j} \in\left[a_{46}^{j} \pm 0.0107\right]$ for $j=1, \ldots, N$. This uncertainty will be taken into account when generating the set of output behaviors using the interval LPV observer.

The proposed observer design procedure was applied to obtain $L_{j}$ such that the poles are in disk LMI region with the parameters $a=-0.5$ and $r=0.5$. In the design of the observer gains $L_{j}$ uncertainty in matrix $A_{j}\left(\vartheta^{j}\right)$ has been considered.

\section{Fault scenarios}

The fault scenarios were implemented in nonlinear TRMS (see equations in [20]) using interval LPV observer designed in the previous section. Figs. 2-3 present the results and the fault detection indicator is presented at the bottom of each graph.

1) Fault scenario 1: An additive sensor fault of the input voltage of the tail motor $f_{u_{t}}$ is defined as:

$$
f_{u_{t}}(t)=\left\{\begin{array}{cl}
0, & \text { for } \quad t<100 \\
0.01, & \text { for } \quad t \geq 100
\end{array}\right.
$$

Fig. 2(a) shows that the azimuth angle of beam $\theta_{h}$ and the estimation given by the interval LPV observer (7) present very close behavior. The prediction bounds of the azimuth angle of beam $\theta_{h}$ are obtained using zonotopes (see Section III) and taking into account the uncertainty in the parameters. The zoom in the figure shows in detail the bounds size. The fault detection test (12) detects the fault at the time $t=$ $100.5 \mathrm{~s}$ and the alarm keeps constant until $t=115.3 \mathrm{~s}$.

Fig. 2(b) shows the pitch angle of beam $\theta_{v}$ with the prediction provided by the interval LPV observer (7). The bounds of pitch angle of beam $\theta_{v}$ are obtained using the zonotope algorithm. In this case, the fault detection test (12) shows that the fault does not affect this angle.

Fig. 2(c) shows the residual signal for azimuth angle of beam $\theta_{h}$ and the envelopes computed using the zonotope method. The envelopes of the residual are adapted following the changes in the system dynamics. The fault detection test (14) shows that the fault alarm is activated in $t=100.5 \mathrm{~s}$.

2) Fault scenario 2: An additive sensor fault of the azimuth angle of beam $f_{\theta_{h}}$ is defined as:

$$
f_{\theta_{h}}(t)=\left\{\begin{array}{cl}
0, & \text { for } \quad t<100 \\
0.03, & \text { for } \quad t \geq 100
\end{array}\right.
$$

Fig. 3(a) shows that the azimuth angle of beam $\theta_{h}$ and the estimation given by the interval LPV observer (7) present a very close response. The fault detection test (12) detects the fault in the time $t=100.025 \mathrm{~s}$ and the alarm keeps constant until $t=101.5 \mathrm{~s}$. This fault $f_{\theta_{h}}$ does not affect the pitch angle of beam $\theta_{v}$ and due to the lack of space has not been included. 


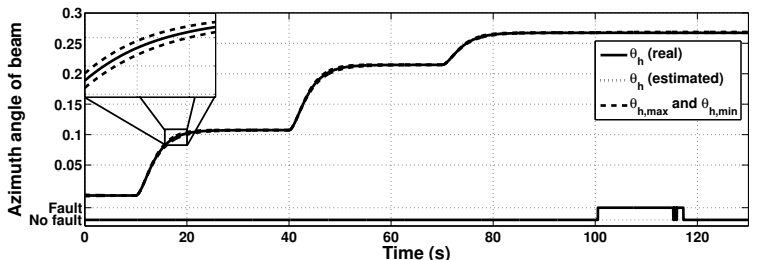

(a)

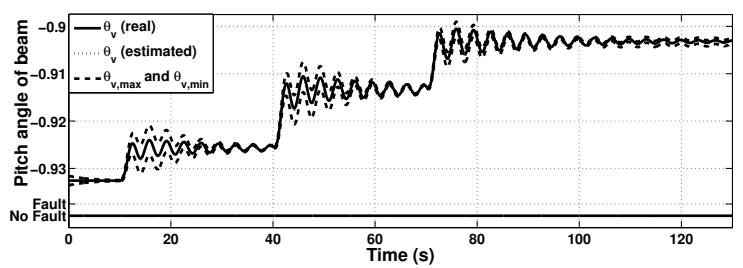

(b)

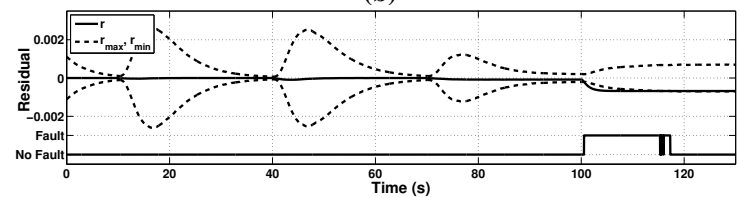

(c)

Fig. 2: (a) Azimuth angle of beam in presence of fault $f_{u_{t}}$. (b) Pitch angle of beam in presence of fault $f_{u_{t}}$. (c) Residual of azimuth angle of beam in presence of fault $f_{u_{t}}$

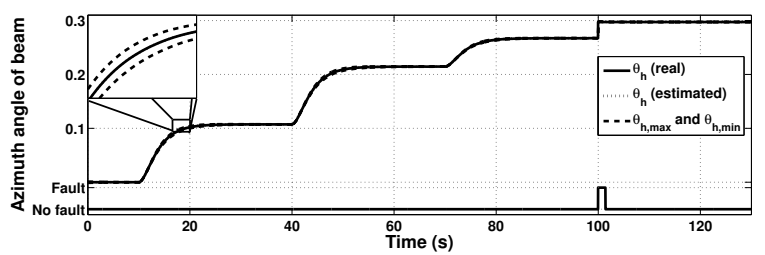

(a)

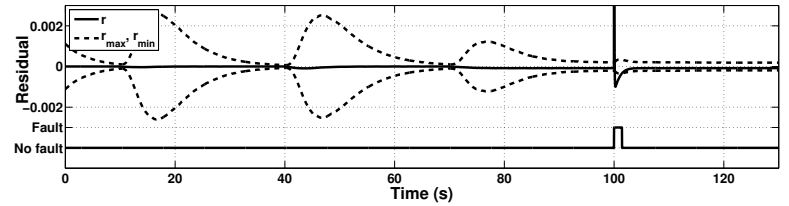

(b)

Fig. 3: (a) Azimuth angle of beam in presence of fault $f_{u_{t}}$. (b) Residual of azimuth angle of beam in presence of fault $f_{\theta_{h}}$.

Fig. 3(b) shows the residual signal for azimuth angle of beam $\theta_{h}$ and its envelopes that are based on zonotope algorithm. The fault detection test (14) shows that the fault alarm is activated at the same time as in Fig. 3(a).

\section{CONCLUSIONS}

In this paper, a robust fault detection using interval LPV observer and zonotopes has been proposed. The gain of the interval LPV observer has been designed using LMI formulation. This method guarantees the pole placement of the observer for each vertex with uncertainties. As a result a set of gains is obtained and these are interpolated to calculate the gain of interval LPV observer (7). A set of estimated outputs based on propagating the uncertainty using zonotopes is proposed. This set has been used to implement the fault detection test. Finally, a TRMS has been used as a case study. It has been described by means of LPV model with uncertainties, which were considered unknown but bounded by intervals. According to the results obtained in the considered fault scenarios, the proposed fault detection approach has been successfully applied.

\section{ACKNOWLEDGMENTS}

This work has been partially funded by the grant CICYT DPI2008-01996 of Spanish Ministry of Education, the by Research Comission of the Generalitat de Catalunya (group SAC ref. 2005SGR00537) and by a grant from Consejo Nacional de Ciencia y Tecnologia (CONACyT), México.

\section{REFERENCES}

[1] J. Chen and R. J. Patton, Robust Model-based Fault Diagnosis for Dynamic Systems. Kluwer Academic Publishers, 1999.

[2] J. Shamma and J. Cloutier, "Gain Scheduled Missile Autopilot Design using Linear Parameter Varying Transformations," AIAA Journal of Guidance, Control, and Dynamics, vol. 16, no. 2, pp. 256-263, 1993.

[3] M. Andrés and G. J. Balas, "Development of Linear-ParameterVarying Models for Aircraft," Journal of Guidance, Control and Dynamics, vol. 27, no. 2, pp. 218-228, 2004.

[4] J. Bokor, Z. Szabo, and G. Stikkel, "Failure Detection for Quasi LPV Systems," Proceedings of the 41st IEEE Conference on Decision and Control, vol. 3, pp. 3318-3323, 2002.

[5] M. Witczak, J. Korbicz, and R. Patton, "A Bounder-error Approach to Designing Unknown Input Observers," in IFAC World Congress (b'02), Barcelona, Spain, 2002.

[6] D. Maksarov and J. Norton, "State Bounding with Ellipsoidal Set Description of the Uncertainty," International Journal of Control, vol. 65 (5), pp. $847-866,1996$.

[7] B. Rinner and U. U. Weiss, "Online Monitoring by Dynamically Refining Imprecise Models," IEEE Transactions on Systems, Man and Cybernetics: Part B, vol. 34 (4), pp. 1811 - 1822, 2004.

[8] M. Milanese, J. Norton, H. Piet-Lahanier, and E. Walter, Eds., Bounding Approaches to System Identification. Springer, 1996.

[9] P. Apkarian, P. Gahinet, and G. Becker, "Self-scheduled $H_{\infty}$ Control of Linear Parameter-Varying Systems: A Design Example," Automatica, vol. 31, no. 9, pp. 1251 - 1261, 1995.

[10] R. Murray-Smith and T. A. Johansen, Multiple Model Approaches to Modelling and Control. Taylor and Francis, 1997.

[11] P. Baranyi, D. Tikk, Y. Yam, and R. J. Patton, "From Differential Equations to PDC Controller Design via Numerical Transformation," Computers in Industry, Elsevier Science, vol. 51, pp. 281-297, 2003.

[12] V. Puig, J. Quevedo, and T. Escobet, "Robust Fault Detection Approaches using Interval Models," in IFAC World Congress (b'02), Barcelona, Spain, 2002.

[13] T. Alamo, J. Bravo, and E. Camacho, "Guaranteed State Estimation by Zonotopes," Automatica, vol. 41, no. 6, pp. 1035-1043, 2005.

[14] M. Chilali and P. Gahinet, " $H_{\infty}$ Design with Pole Placement Constraints: An LMI Approach," IEEE Transactions on Automatic Control, vol. 41, no. 3, pp. 358-367, 1996.

[15] M. Rodrigues, D. Theilliol, and D. Sauter, "Design of an Active Fault Tolerant Control and Polytopic Unknown Input Observer for Systems described by a Multi-Model Representation," 44th IEEE Conference on Decision and Control and European Control Conference ECC, Sevilla, Spain (CD-ROM), 2005.

[16] W. Kuhn, "Rigorously Computed Orbits of Dynamical Systems without the Wrapping Effect," Computing, vol. 61, no. 1, pp. 47-67, 1998.

[17] R. Moore, Interval Analysis. Prentice Hall, 1966.

[18] C. Combastel, "A State Bounding Observer based on Zonotopes," in European Control Conference, 2003.

[19] B. Bamieh and L. Giarré, "Identification of Linear Parameter Varying Models," International Journal Robust Nonlinear Control, vol. 2, no. 12, pp. 841-853, 2002.

[20] Twin Rotor MIMO System Advanced Teaching Manual 1 (33-0074M5), Feedback Instruments Ltd, Crowborough, UK., 1998. 